\title{
Origin of the enhanced structural and reorientational relaxation rates in the presence of relatively weak dc electric fields*
}

\author{
Alice Vegiri ${ }^{\ddagger}$ \\ National Hellenic Research Foundation, Institute of Theoretical and Physical \\ Chemistry, 48 Vas. Constantinou Ave., Athens 11 635, Greece
}

\begin{abstract}
The origin of the dramatic increase of the reorientational and structural relaxation rates of single water molecules in clusters of size $N=16,32$, and 64 at $T=200 \mathrm{~K}$, under the influence of an external, relatively weak electric field $\left(\sim 0.510^{7} \mathrm{~V} / \mathrm{cm}\right)$ is examined through molecular dynamics simulations. The observed effect is attributed not to any profound structural changes, but to the increase of the size of the molecular cage. The response of water to an electric field in this range shows many similarities with the dynamics of water under low pressure. By referring to simulations and experiments from the literature, we show that in both cases the observed effects are dictated by a common mechanism.
\end{abstract}

\section{INTRODUCTION}

In our earlier work [1-4], where the structural and dynamical effects of an external dc electric field on cold $(T=200 \mathrm{~K})$ water clusters have been thoroughly examined, we observed a dramatic decrease of reorientational and single-particle density relaxation times when the field was switched on. The decrease (by as much as almost 10 times) was more intense for reorientational relaxations compared to density relaxations, and it was equally observed for the dipole vector, the vector joining the two hydrogen atoms, and the vector perpendicular to the molecular plane. When the electric field was further increased to values approaching the first transition field $E_{\mathrm{c} 1}=1.510^{7} \mathrm{~V} / \mathrm{cm}$, where a structural transition to a dipole-aligned state takes place, relaxation times were observed to increase again. A similar trend was also followed by the self-diffusion coefficient. In addition, the examination of the low-field selfvan Hove correlation functions, which give the probability of finding a particle at a position $r$, at time $t$ given that the same particle was at $r=0$ at $t=0$, displayed a Gaussian behavior, indicative of the liquid character the samples acquire at low fields. The origin of this effect could not be explained by relying on structural changes and on energetics alone, since atom pair-correlation functions and molecular pair energies were identical with the no-field case for fields less than $E_{\mathrm{c} 1}$ where this phenomenon was observed. An explanation was given by taking into account the additional torques, which are exerted on molecules from the electric field and which enhance the reorientational motions, confronting in this way any steric hindrances. The resultant increased rotational mobility would facilitate in turn molecular translations, through rotational-translational coupling.

However, when the electric field becomes stronger than $E_{\mathrm{cl}}$, the dependence of the relaxation rates on the field values, (decreasing rates with increasing field) can be understood by considering the corresponding structural changes, which are dictated primarily by the necessity of the dipoles to orient

\footnotetext{
*Lecture presented at the European Molecular Liquids Group (EMLG) Annual Meeting on the Physical Chemistry of Liquids: Novel Approaches to the Structure, Dynamics of Liquids: Experiments, Theories, and Simulation, Rhodes, Greece, 7-15 September 2002. Other presentations are published in this issue, pp. 1-261.

FTel: +30-210-7273 803; Fax: +30-210-7273 794; E-mail: avegiri@eie.gr
} 
themselves parallel to the field and remain as far as possible from each other in order to reduce their mutual repulsions.

The dynamic response of supercooled water to a relatively weak electric field shares many similarities with water when external pressure, in the few kbar range, is applied, and for conditions that are not adequate to induce structural transitions to one of the ice forms that are known to emerge under much higher values of pressure. In this case, reorientational relaxation rates and self-diffusion coefficients have also been found, both in experiments [5,6] and in simulations [7-11] to go through a maximum with increasing pressure and then reduce with further pressure increase. Moreover, it was found that this effect was more intense in the supercooled regime, and that reorientations rather than density relaxations were more severely affected [5], as is exactly the case with electric fields as well. The prevailing explanation for this kind of response, which is counterintuitive and at odds to what is normally expected in simple, unconnected liquids, where diffusion decreases monotonically with increasing density and pressure, is that there is a change in the local structure with the appearance of a fifth or even a sixth molecule in the coordination sphere of an otherwise tetrahedrally coordinated water molecule [12]. The distortion of the $\overline{O O O}$ angle away from the tetrahedral arrangement has as a result the weakening of the hydrogen bonding.

If we take a closer look at the experimental measurements concerning the self-diffusion coefficient as a function of pressure [5,6], we observe that it starts increasing after a low pressure is applied and that the maximum is reached for a pressure value equal to about $1.5 \mathrm{kbar}$ and for temperatures $T=243 \mathrm{~K}$ [5] or $T=277 \mathrm{~K}$ [6]. Regarding structural information, oxygen-oxygen pair-correlation functions have been derived through X-ray scattering in liquid water at $T=293 \mathrm{~K}$ for pressures up to $7.7 \mathrm{kbar}$ [13]. In these experiments, a pronounced reduction of the intensity of the second neighbors' shell at $4.5 \AA$ and the appearance of an additional peak at $\sim 3.3 \AA$ were observed at a pressure of about $4 \mathrm{kbar}$, which, according to previous work [5-11] is higher than the pressure where the maximum of the diffusion coefficient and of the reorientational relaxation rates is obtained. The $4.5 \AA$ peak corresponds to the distance between molecules residing at the vertices of a regular tetrahedron, whereas the additional peak at $3.3 \AA$, which is met in different forms of dense ice polymorphs is attributed to interstitial molecules. In this respect, it seems that the observed increase of the diffusion constant and reorientational relaxation rates in the low-pressure regime cannot be directly associated with pronounced structural changes in the liquid. Such structural changes indeed occur at higher pressures, but they act to the direction of retarding relaxation rather than enhancing it.

Given the similarities in dynamics of water under relatively weak pressures and electric fields at low temperatures, in the present work we examine whether the underlying structural factors may be responsible for the observed effects. As in our earlier work [3,4], we carry out molecular dynamics simulations in the microcanonical ensemble at a temperature of about $200 \mathrm{~K}$ for water clusters with size $N=16, N=32$, and $N=64$ and for electric fields less than $E_{\mathrm{c} 1}=1.510^{7} \mathrm{~V} / \mathrm{cm}$. Details about the potential and computational method will not be repeated here.

From the examination of the computed atom pair-correlation functions, the distribution of the angles formed by all triads of neighboring oxygen atoms, and the distribution of the potential energies of molecular pairs, not any indication of a structural change at low fields responsible for the observed dynamical effect was detected. Certainly, low pressure or alternatively a weak electric field induce a loosening of the molecular cage (otherwise, the molecules could not be able to translate and rotate more freely), but this effect should be of dynamical rather than of structural origin. In fact, at these low electric fields, an enhancement of the molecular mean vibrational amplitude was observed, which displayed with the field a similar trend as the relaxation times and the diffusion coefficients [4]. Finally, we should like to note that the observations concerning water clusters could not be directly extrapolated to the bulk, because of the surface effects inherent in clusters. However, behaviors observed in small systems could serve as a guideline for the mechanisms that may be operating in extended systems as well. 


\section{RESULTS AND DISCUSSION}

As stated in the introduction, the application of a relatively weak electric field on a cold water cluster had as a result the dramatic decrease of the reorientational and single-particle density relaxation times. The corresponding relaxation times are displayed in Table 1 for all cluster sizes examined, i.e., $N=16$, $N=32$, and $N=64$ and for the fields $E=0, E=0.510^{7} \mathrm{~V} / \mathrm{cm}$, and $E=110^{7} \mathrm{~V} / \mathrm{cm}$.

Table 1 Effective reorientational and structural relaxation times in ps at $T=200 \mathrm{~K}$. Field values are in $10^{7} \mathrm{~V} / \mathrm{cm}$.

\begin{tabular}{|c|c|c|c|}
\hline \multicolumn{4}{|c|}{$\tau^{H H}$} \\
\hline$N$ & 16 & 32 & 64 \\
\hline$E=0$ & 26.2 & 30.5 & 50.2 \\
\hline$E=0.5$ & 3.1 & 5.8 & 22.2 \\
\hline$E=1$ & 6.9 & 56.5 & 45.8 \\
\hline \multicolumn{4}{|c|}{$\tau^{\mu}$} \\
\hline$N$ & 16 & 32 & 64 \\
\hline$E=0$ & 41 & 41.8 & 73.4 \\
\hline$E=0.5$ & 3.8 & 11.2 & 28.2 \\
\hline$E=1$ & 8.8 & 55.5 & 50.9 \\
\hline \multicolumn{4}{|c|}{$\tau^{\mathrm{SISF}-z} Q=2.2 \AA^{-1}$} \\
\hline$N$ & 16 & 32 & 64 \\
\hline$E=0$ & 12.5 & 7.1 & 14.2 \\
\hline$E=0.5$ & 2.1 & 4.2 & 3.2 \\
\hline$E=1$ & 3.3 & 5.2 & 11.7 \\
\hline
\end{tabular}

$\tau^{H H}$ and $\tau^{\mu}$ denote the reorientational relaxation times of the unit vector $\boldsymbol{\mu}$, joining the hydrogen atoms of a water molecule and of the dipole unit vector $\boldsymbol{H H}$, respectively. They are derived from the corresponding single particle correlation functions defined as

$$
\begin{array}{lrl}
C^{H H}(t)=\langle\boldsymbol{H} \boldsymbol{H}(t) \cdot \boldsymbol{H H}(0)\rangle & C^{H H}(t) \rightarrow 0 \text { as } t & \rightarrow \infty \\
C^{\mu}(t)=\langle[\boldsymbol{\mu}(t)-\langle\cos \theta\rangle] \cdot[\boldsymbol{\mu}(0)-\langle\cos \theta\rangle]\rangle & \text { so that } C^{\mu}(t) \rightarrow 0 \text { as } t \rightarrow \infty
\end{array}
$$

where $\langle\cos \theta\rangle$ is the average angle the dipoles form with the field axis directed along the $z$ laboratory axis.

$\tau^{\mathrm{SISF}-z}(Q)$ denotes the structural relaxation time at a particular wavevector $\mathrm{Q}$ and is taken from the incoherent, self-intermediate scattering function along the electric field direction, (SISF-z).

$$
\operatorname{SISF}(Q, t)=\frac{1}{N} \sum_{i}^{N}\left\langle\exp \left\{i \boldsymbol{Q}\left[\boldsymbol{r}_{i}(t)-\boldsymbol{r}_{i}(0)\right]\right\}\right\rangle
$$

The long time part of the aforementioned correlation functions was fitted to a stretched exponential function as described in refs. [3,4,14].

By inspecting Table 1, we observe that the influence of the external field is stronger on the reorientational relaxation times, the same way it was observed in the case of external pressure [5]. Also, the dipole relaxation times, which are associated with tumbling molecular motions, are larger than the $\boldsymbol{H} \boldsymbol{H}$ relaxation times, which are associated with spinning motions, as is the case in normal liquid water and other liquids as well $[15,16]$ at zero field. 
In order to elucidate the mechanism responsible for this dynamical response, we examined whether the application of a weak field induces observable structural changes in the clusters. In this respect, we calculated oxygen-oxygen pair-correlation functions (illustrated in Fig. 1), distributions of the $\overline{O O O}$ angles formed by three neighboring oxygen atoms (displayed in Fig. 2), and distributions of water-water pair-interaction energies (displayed in Fig. 3). The electric field values are indicated on the corresponding curves in Figs. 1-3.

Regarding the pair-correlation functions (Fig. 1), they appear to be almost identical to each other for fields less than $E_{\mathrm{cl}}$, by retaining the same shell structure as for $E=0$. A profound change, however, is observed, as expected, for $E=E_{\mathrm{c} 1}=1.510^{7} \mathrm{~V} / \mathrm{cm}$, according to our previous findings $[3,4]$.

Similarly, by inspecting Figs. 2 and 3 , we observe that the distributions for $E=0$ and $E=0.510^{7} \mathrm{~V} / \mathrm{cm}$ are indistinguishable, indicating that it is not the change in structure that induces the faster dynamics. Notice from Table 1 that the drop in relaxation times is quite intense for $E=0.510^{7} \mathrm{~V} / \mathrm{cm}$. In these figures, the corresponding distributions for fields equal to and stronger than $E_{\mathrm{c} 1}$ are also included for comparison. The structural changes for $E \geq E_{\mathrm{c} 1}$ have been examined in detail in $[3,4]$. The two pronounced peaks observed in the $\overline{O O O}$ angle distribution at about $90^{\circ}$ and $123^{\circ}$ correspond to squares and pentagonal rings, respectively. As the field gets stronger, the system transforms to proton-ordered cubic structures, a process known as electrofreezing [17], and as a consequence the peak corresponding to squares is enhanced relatively to the peak that corresponds to pentagons.

The low-energy band (energies less than $-2.5 \mathrm{kcal} / \mathrm{mol}$ ) in the distributions of the molecular pair interaction energies in Fig. 3, represents hydrogen-bonded neighbors, while the peak near $0 \mathrm{kcal} / \mathrm{mol}$ includes all distant molecules. As the electric field increases beyond $E_{\mathrm{c} 1}$, the low-energy peak shifts to smaller values, indicating the weakening of the hydrogen bond at strong fields.

From the arguments presented above, regarding the influence of the structural factor on dynamics, we may infer that at low fields the molecular environment remains unaltered as at $E=0$ and that the reason for the enhanced relaxation should lie elsewhere.

In a previous work regarding the $N=32$ cluster at $T=200 \mathrm{~K}$ [4], we had found that the mean vibrational amplitude of a single water molecule in its cage substantially increases at low fields. For fur-
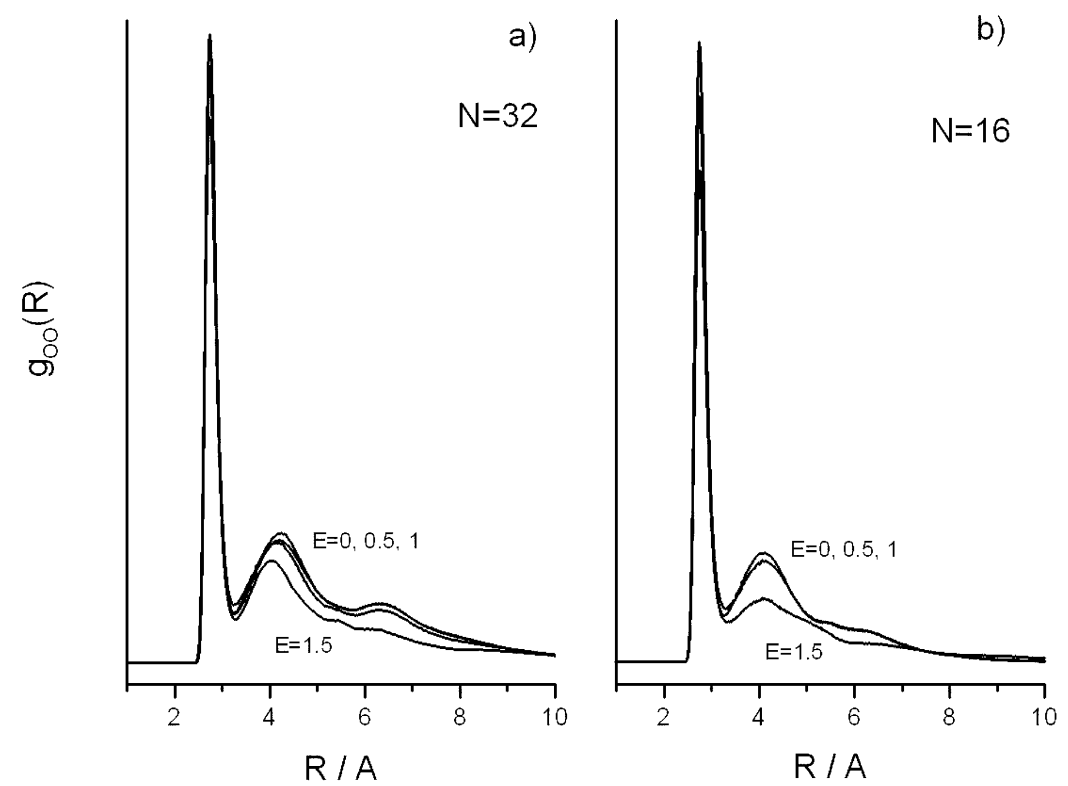

Fig. 1 Oxygen-oxygen pair-correlation functions for $E=0, \mathrm{E}=0.510^{7} \mathrm{~V} / \mathrm{cm}, E=110^{7} \mathrm{~V} / \mathrm{cm}$, and $E=1.510^{7}$ V/cm. (a) $N=32$, (b) $N=16$. 


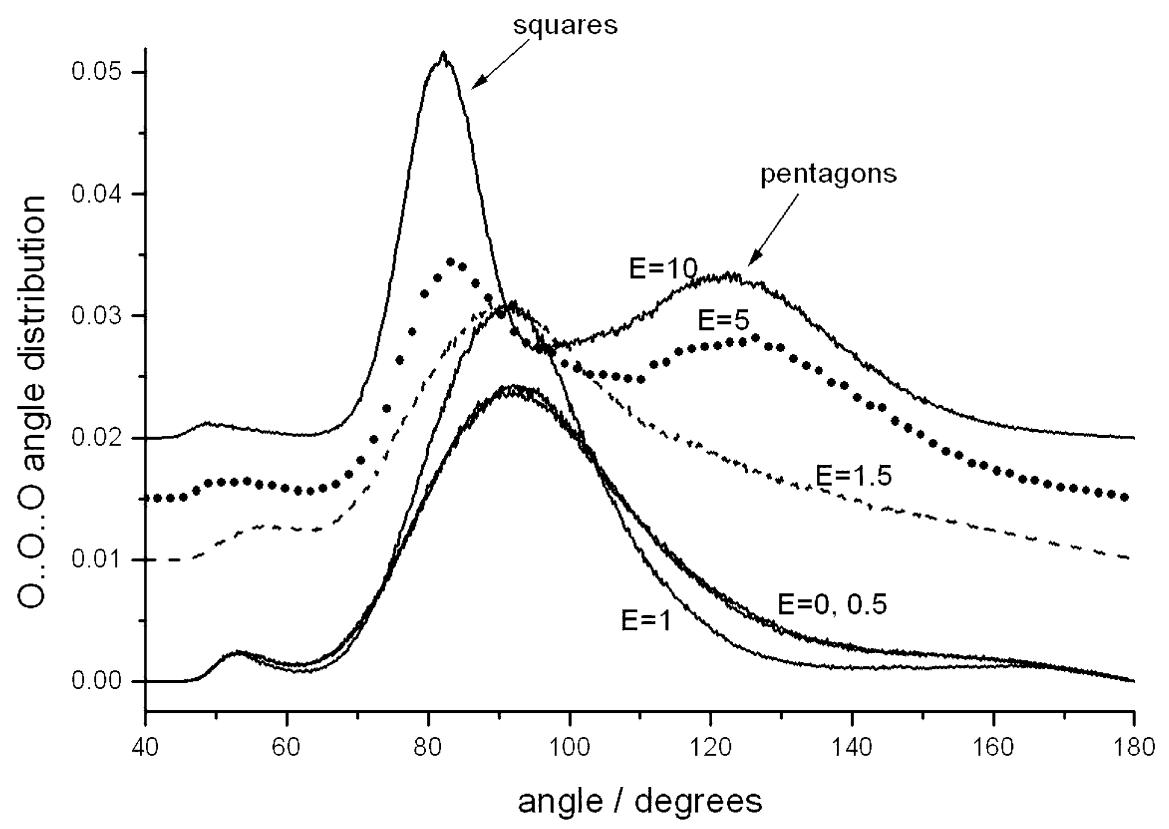

Fig. 2 O..O..O angle distribution for $N=16$. Electric field values are in $10^{7} \mathrm{~V} / \mathrm{cm}$. The curves corresponding to $E=1.510^{7} \mathrm{~V} / \mathrm{cm}, E=510^{7} \mathrm{~V} / \mathrm{cm}$, and $E=1010^{7} \mathrm{~V} / \mathrm{cm}$ are vertically displaced for clarity.

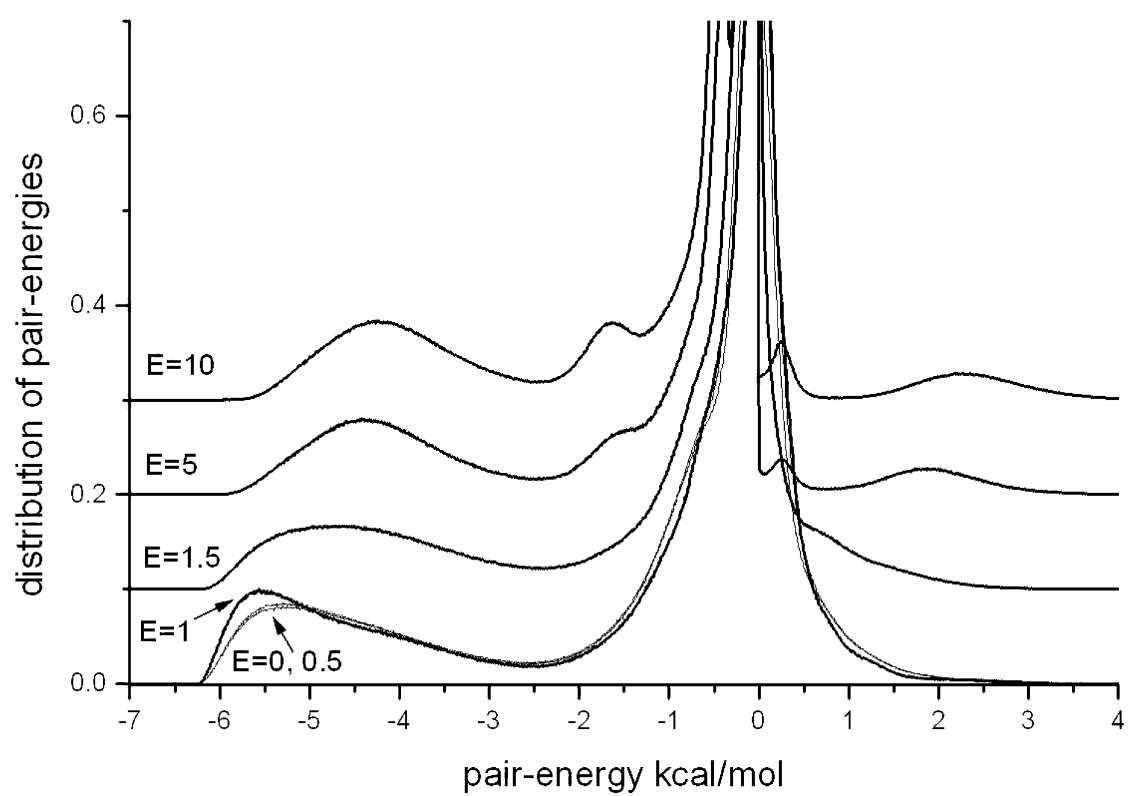

Fig. 3 Distribution of water-water interaction energies for $N=16$. Electric field values are in $10^{7} \mathrm{~V} / \mathrm{cm}$. The curves corresponding to $E=1.510^{7} \mathrm{~V} / \mathrm{cm}, E=510^{7} \mathrm{~V} / \mathrm{cm}$, and $E=1010^{7} \mathrm{~V} / \mathrm{cm}$ are vertically displaced by 0.1 for clarity.

ther field increase the mean vibrational amplitude was found to decrease again. Mean vibrational amplitudes at a particular field are derived from the corresponding self-intermediate scattering functions calculated at several wavevectors through a procedure described in refs. [4,14]. 
In the present work, we repeat the calculations for a smaller, $N=16$ and a larger, $N=64$ cluster, from where the trend observed initially for $N=32$ is reproduced. The results are displayed in Fig. 4 as a function of the electric field. Indeed, as the field is switched on, at $E=0.510^{7} \mathrm{~V} / \mathrm{cm}$, an increase of the molecular vibrational amplitude is observed, which is consistent with the loosening of the molecular cage, known to exist at these low temperatures. Therefore, since each molecule is a part of a nearby cage meant for another neighbor, the entire structure is loosened, but not destroyed. We believe that this gives a physical explanation for the enhanced rotational and structural dynamics of the molecules at low electric fields.

Interestingly enough, a cage size increase was also calculated in water for the same range of pressures for which relaxation times were found to go through a minimum [10].

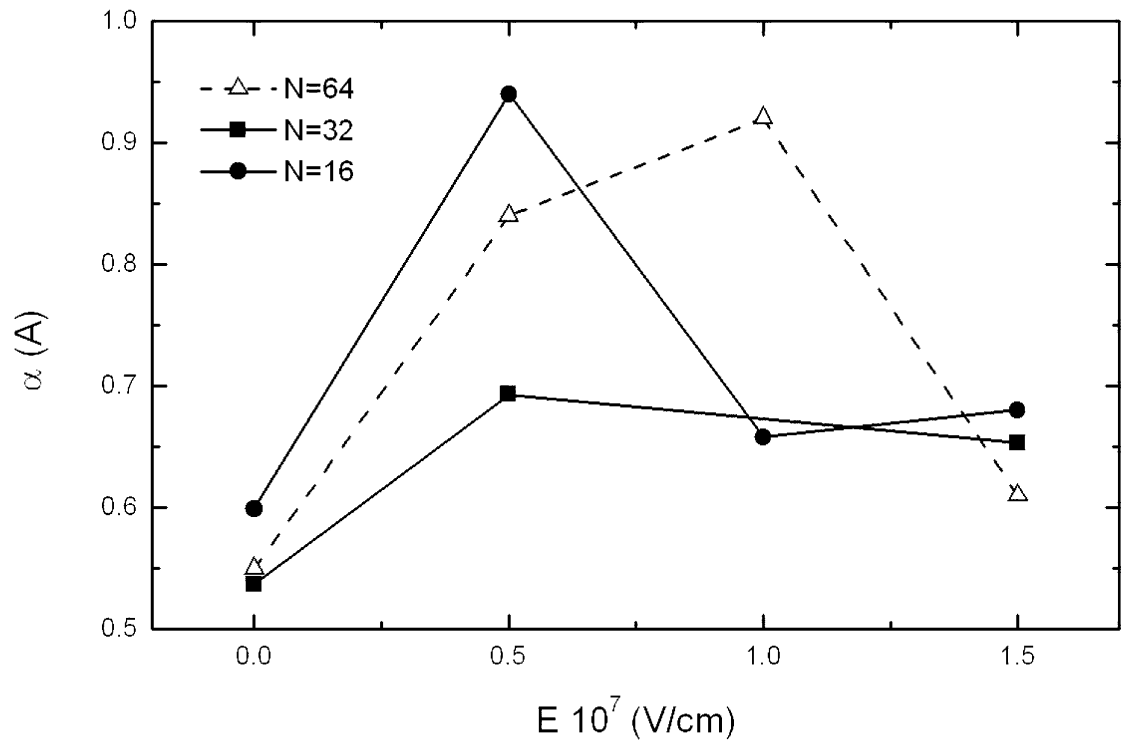

Fig. 4 Water molecule mean vibrational amplitude as a function of the electric field, for $N=16, N=32$, and $N=64$.

\section{CONCLUSIONS}

In this work, we attempt to trace the origin of the enhanced reorientational and structural dynamics observed in cold water clusters at low electric fields. This effect is attributed not to structural changes, but to the increase of the vibrational amplitude of single water molecules inside their cages.

A similar behavior is also displayed by liquid water in the supercooled regime when low pressure is applied. Namely, for low pressures reorientational and structural relaxation times have been found to go though a minimum, whereas at the same time an increase of the mean molecular vibrational amplitude was observed.

We have shown that water responds in the same way to an external force field, be it pressure or electric field, provided that both are of such strength so as not to induce substantial structural changes. Before making any generalizations however, it would be instructive to extend our investigations to other kinds of external fields and to the bulk. Calculations regarding the influence of dc electric fields on bulk water are currently underway.

\section{ACKNOWLEDGMENTS}

Financial support by a grant from INTAS (99-01162) is gratefully acknowledged. 


\section{REFERENCES}

1. S. V. Shevkunov and A. Vegiri. J. Mol. Struct. (Theochem) 574, 27 (2001).

2. S. V. Shevkunov and A. Vegiri. J. Mol. Struct. (Theochem) 593, 19 (2002).

3. A. Vegiri and S. V. Shevkunov. J. Chem. Phys. 115, 4175 (2001).

4. A Vegiri. J. Chem. Phys. 116, 8786 (2002).

5. F. X. Prielmeier, E. W. Lang, R. J. Speedy, H.-D. Ludemann. Phys. Rev. Lett. 59, 1128 (1987).

6. L. A. Wolf. J. Chem. Soc. Farad. Trans. I 71, 784 (1975).

7. K. Bagchi, S. Balasubramanian, M. L. Klein. J. Chem. Phys. 107, 8561 (1997).

8. M. R. Reddy and M. Berkowitz. J. Chem. Phys. 87, 6682 (1987).

9. F. W. Starr, S. Harrington, F. Sciortino, H. E. Stanley. Phys. Rev. Lett. 82, 3629 (1999).

10. F. W. Starr, F. Sciortino, H. E. Stanley. Phys. Rev. E 60, 6757 (1999).

11. F. W. Starr, M.-C. Bellisent-Funel, H. E. Stanley. Phys. Rev. E 60, 1084 (1999).

12. P. A. Netz, F. Starr, M. C. Barbosa, H. E. Stanley. J. Mol. Liq. 101, 159 (2002).

13. A. V. Okhulkov, Yu. N. Demianets, Yu. E. Gorbaty. J. Chem. Phys. 100, 1578, (1994).

14. S. H. Chen, C. Liao, F. Sciortino, P. Gallo, P. Tartaglia. Phys. Rev. E 59, 6708 (1999).

15. R. W. Impey, P. A. Madden, I. R. McDonald. Mol. Phys. 46, 513 (1982).

16. H. J. Böhm, R. M. Lynden-Bell, P. A. Madden, I. R. McDonald. Mol. Phys. 51, 761 (1984).

17. I. M. Svishchev and P. G. Kusalik. Phys. Rev. Lett. 73, 975 (1994). 